\title{
Seminario Taller del GDRI «APOCAMO»: Estado y movimiento indígena en las tierras bajas de los países andinos
}

Lima, 17 al 19 de septiembre de 2014

\section{Alexandre Surrallés}

\section{(2) OpenEdition}

12 Journals

\section{Edición electrónica}

URL: http://journals.openedition.org/bifea/6013

DOI: $10.4000 /$ bifea.6013

ISSN: 2076-5827

Editor

Institut Français d'Études Andines

\section{Edición impresa}

Fecha de publicación: 1 diciembre 2014

Paginación: 654-659

ISSN: 0303-7495

\section{Referencia electrónica}

Alexandre Surrallés, «Seminario Taller del GDRI «APOCAMO»: Estado y movimiento indígena en las tierras bajas de los países andinos », Bulletin de l'Institut français d'études andines [En línea], 43 (3) I 2014, Publicado el 08 diciembre 2014, consultado el 06 noviembre 2020. URL : http:// journals.openedition.org/bifea/6013; DOI : https://doi.org/10.4000/bifea.6013

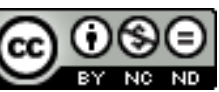

Les contenus du Bulletin de l'Institut français d'études andines sont mis à disposition selon les termes de la licence Creative Commons Attribution - Pas d'Utilisation Commerciale - Pas de Modification 4.0 International. 


\section{SEMINARIO TALLER DEL GRDI «APOCAMO»1: ESTADO Y MOVIMIENTO INDÍGENA EN LAS TIERRAS BAJAS DE LOS PAÍSES ANDINOS}

La Paz, 16-18 de octubre del 2014

En vistas a conseguir espacios de reconocimiento de sus derechos, participación y representación, el movimiento indígena amazónico en los países andinos se ha organizado desde la década de los años 1980, en parte como una plataforma para interactuar con el Estado. Sin embargo, el Estado es un ente complejo y heterogéneo. Es por supuesto el Estado central, en su variedad de poderes y ministerios, pero también son los gobiernos regionales, provinciales y locales muy importantes para las poblaciones indígenas amazónicas, alejadas de las grandes capitales. Estos diferentes niveles y estamentos imponen la práctica burocrática como lengua de contacto, que las organizaciones indígenas mimetizan primero, reproducen después, e incorporan en sus relaciones con los demás.

Si el Estado es polifacético, las relaciones que el movimiento indígena puede establecer con él, son también múltiples y variadas. Tres ejemplos. El brazo electoral del movimiento indígena, que postula en los diversos comicios para elegir autoridades locales, regionales y nacionales. Los profesionales indígenas

1 Grupo de Investigación internacional (GDRI) Apocamo «Antropología política contemporánea en la Amazonía occidental» (IFEA, CNRS, EHESS, UNAL, PUCP, Flacso). 


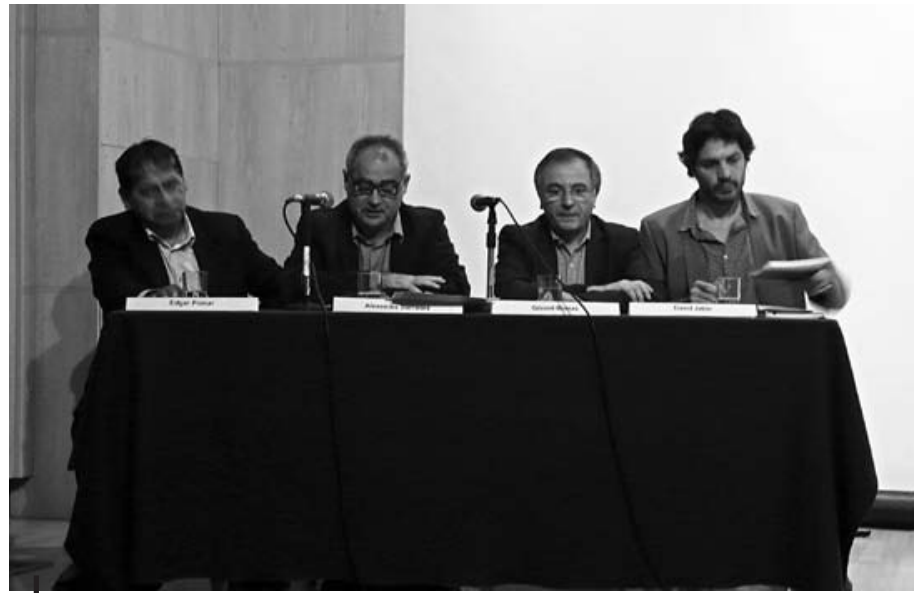

Mesa de presentación del seminario Apocamo en el Musef, La Paz (C) Jean-Pierre Chaumeil en los ámbitos sectoriales de salud, educación o medio ambiente, a nivel local y regional. Un ejemplo más: los dirigentes o ex dirigentes indígenas ejerciendo como altos funcionarios del Estado central.

El movimiento indígena, con esta política de relación con el Estado, puede pensar que lo influye. Pero puede ser también el Estado el que a través de esta relación controle el movimiento indígena, para usarlo para sus fines o disolver su fuerza. En este contexto, es interesante preguntarse por el

significado de la autonomía como política indígena en el área amazónico-andina, difundida por todo el continente, cuando abandona la interlocución con el Estado y dedica las energías a organizarse al margen. Se hace necesario también analizar la relación de las autoridades electas indígenas locales, regionales y nacionales, con el movimiento indígena y con el resto de la población no indígena.

Con la finalidad de explorar estos temas, el sexto seminario-taller del grupo Apocamo tuvo lugar los pasados días 16 y 17 de octubre de 2014, en los locales del Museo Nacional de Etnografía y Folklore, Musef, en La Paz, Bolivia. Las palabras de bienvenida fueron ofrecidas por el Sr. Edgar Pomar, responsable de las Relaciones Públicas del Musef y el Sr. Gérard Borras, director del IFEA (Umifre 17 CNRS-MAEDI USR 3337 América Latina). Acto seguido los organizadores de este seminario taller David Jabin (Université Paris Ouest-EREA/CIHA), y yo mismo, Alexandre Surrallés (CNRS-IFEA), presentaron los objetivos del encuentro y el programa, que se dividía en dos secciones, un día para cada una. Estas secciones fueron tituladas: «Estado y pueblos indígenas, balances por país y comparaciones» y «Poder local, poder estatal, cara a cara».

Tal y como el título indica, el primer día se presentaron cuatro panoramas sobre la historia y la actualidad de las relaciones entre Estado y movimiento indígena, uno por cada país del área andina, dejando a los comentaristas la labor de establecer comparaciones entre los países.

François Correa arrancó con la primera presentación titulada Políticas Estatales y Restricciones a la Autonomía Indígena en la Amazonía Colombiana. El Prof. Correa abordó la discusión sobre la reorganización de los indígenas al interior de los Estados amazónicos que tiende a pendular entre la oposición de formas tradicionales de organización interna y las nuevas formas asociativas surgidas al calor de nuevos movimientos sociales para la defensa de sus derechos colectivos. 
En esta ponencia se analizó dicha situación en la Amazonía colombiana frente a las políticas públicas recientes que apoyan la ampliación de la frontera agropecuaria, la explotación de minerales e hidrocarburos y el desarrollo de la infraestructura vial. Todo ello a la luz de las características de la reorganización del movimiento indígena, las trasformaciones promovidas por el Estado y su participación en las entidades administrativas a través de los comicios electorales. Los comentarios fueron a cargo de Daniela Ricco y Alexandre Surrallés.

Después de un debate plenario, Ana María Lema presentó una ponencia titulada Un Estado intermitente. Las relaciones entre el Estado boliviano y las poblaciones indígenas de tierras bajas, 1825-1990. Esta presentación partía de la constatación que pese a ocupar una importante parte del territorio nacional, las poblaciones indígenas de tierras bajas no han sido objeto de políticas claras por parte del Estado boliviano, desde la creación de la República. Es a medida que se fue conociendo el potencial económico de esta amplia y compleja región que el Estado intentó sentar presencia en la misma, preferentemente recurriendo para ello a mediadores (las misiones, los fortines) o bien a actores externos (colonizadores). Ana María Lema consideró también cómo, paralelamente, los indígenas fueron saliendo de su invisibilización para hacer escuchar su voz, primero a través de rebeliones y luego con la conformación de sus organizaciones indígenas que, poco a poco, empezaron a retar al Estado. Jean-Pierre Chaumeil y David Jabin ofrecieron sendos comentarios antes de un turno abierto de intervenciones.

Por la tarde Fernando García Serrano presentó una comunicación titulada Análisis de la relación Estado y pueblos indígenas ecuatorianos (1990-2013). A partir de la revisión de cinco ámbitos de la relación Estado/pueblos indígenas, esto es, territorialidad, recursos naturales y consulta previa, justicia indígena, bienestar y participación política, el Prof. García Serrano analizó los avances, retrocesos y vaciamientos vividos en esta relación durante el período 1990-2013, con el fin de aportar elementos de contraste con los demás países que viven circunstancias semejantes en América Latina. Según la presentación, especial importancia tiene para el caso ecuatoriano las constituciones de 1998 y 2008, en las cuales se reconoció el carácter pluricultural y pluriétnico del Estado ecuatoriano en la primera, y el carácter plurinacional e intercultural en la segunda. Así mismo, el movimiento indígena desde su surgimiento como actor en la política nacional desde 1990, no solamente ha sido pionero y referente en la región, sino que ha atravesado diversas etapas de auge y crisis que lo convierten en un caso ilustrativo de investigación y discusión. Jorge Komadina y Laetitia Perrier Bruslé proporcionaron algunos alcances comparativos antes de la participación de la audiencia.

Para terminar el día, Oscar Espinosa presentó una ponencia cuyo título, La relación entre el Estado y las sociedades indígenas en la Amazonía peruana: balance histórico, avanzaba los temas desarrollados. En concreto, Espinosa realizó una síntesis de las diferentes visiones que el Estado ha tenido de las poblaciones indígenas amazónicas a lo largo de los últimos decenios. Según Espinosa, estas visiones han considerado hasta muy recientemente que estas poblaciones no existen o que 
no merecen existir. Últimamente se reconoce al fin su existencia, pero solamente a condición de no obstaculizar con reivindicaciones particularistas al llamado «desarrollo» necesario para el país en su conjunto. Es así como se ha ido creando entre las poblaciones indígenas y sus organizaciones un progresivo desafecto hacia el Estado hasta llegar a ser visto incluso como una amenaza después del conflicto de Bagua. François Correa y Andrea-luz Gutiérrez Choquevilca realizaron los comentarios pertinentes y después de un debate plenario se convocó a los asistentes para el día siguiente.

Durante el segundo día, y según estaba previsto, se abordaron algunos estudios de caso particulares, de aspectos varios y de magnitudes diferentes, de la relación entre el poder local y el estatal cuando el movimiento indígena está presente. Fernando García Serrano empezó la jornada con una comunicación titulada Yo voy a ser presidente del país: el ejercicio del poder local desde la diversidad. Marcelino Chumpi, prefecto de la provincia de Morona Santiago, Amazonia ecuatoriana. La ponencia reflexionó sobre el ejercicio del poder local de la prefectura de la provincia de Morona Santiago ejercido por Marcelino Chumpi, militante del Movimiento de Unidad Plurinacional Pachakutik en el período 2008-2013. El análisis se concentró en tres ejes vinculados a la participación y representación política: los procesos electorales de 2008 y 2013; la ejecución del mandato ofrecido durante la campaña y la relación del prefecto con las organizaciones indígenas y no indígenas de la provincia. Los comentarios fueron a cargo de Ely Linares y Ximena Soruco Soluguren antes de dejar paso a las intervenciones de la audiencia.

Andrea-Luz Gutiérrez Choquevilca expuso la comunicación siguiente con el título Traducción y pragmática de las interacciones verbales entre Apus, comunidades y representantes del Estado: el caso de los runa del Pastaza (Provincia Datem de Marañón, Perú). Esta analizó cómo desde el año 2008, los dirigentes quechua del Pastaza han sentado las bases de un nuevo diálogo con el Estado peruano sobre el caso de la explotación petrolera en sus territorios al amparo del Convenio 169 de la OIT. En el marco de una etnogénesis política, mezcla de resistencia y creatividad, los líderes quechua se han apropiado del idioma burocrático con el fin de controlar las mesas de diálogo con el Estado, reclamando la implementación de la consulta previa vigente desde el año 2011, la indemnización y compensación de los daños socioambientales producidos durante más de cuatro décadas de explotación. Sin embargo, constata la ponencia, este diálogo se despliega en varios planos referenciales, solicitando de parte de los dirigentes un esfuerzo constante de traducción y adaptación de las concepciones tradicionales del poder. Esta configuración fue examinada con detalle para mostrar la expresiones del actual «desengaño» político de los runa así como la dimensión de incertidumbre epistemológica que acompaña la construcción de las nuevas formas de legitimidad indígena. Pablo Quisbert y Alexandre Surrallés propusieron algunas reflexiones y preguntas antes del debate plenario.

Por la tarde, Philippe Erikson presentó bajo el título Si matamos a un brasileño, en qué le concierne al gobierno? Análisis de un caso de brujería entre los chacobo, una 


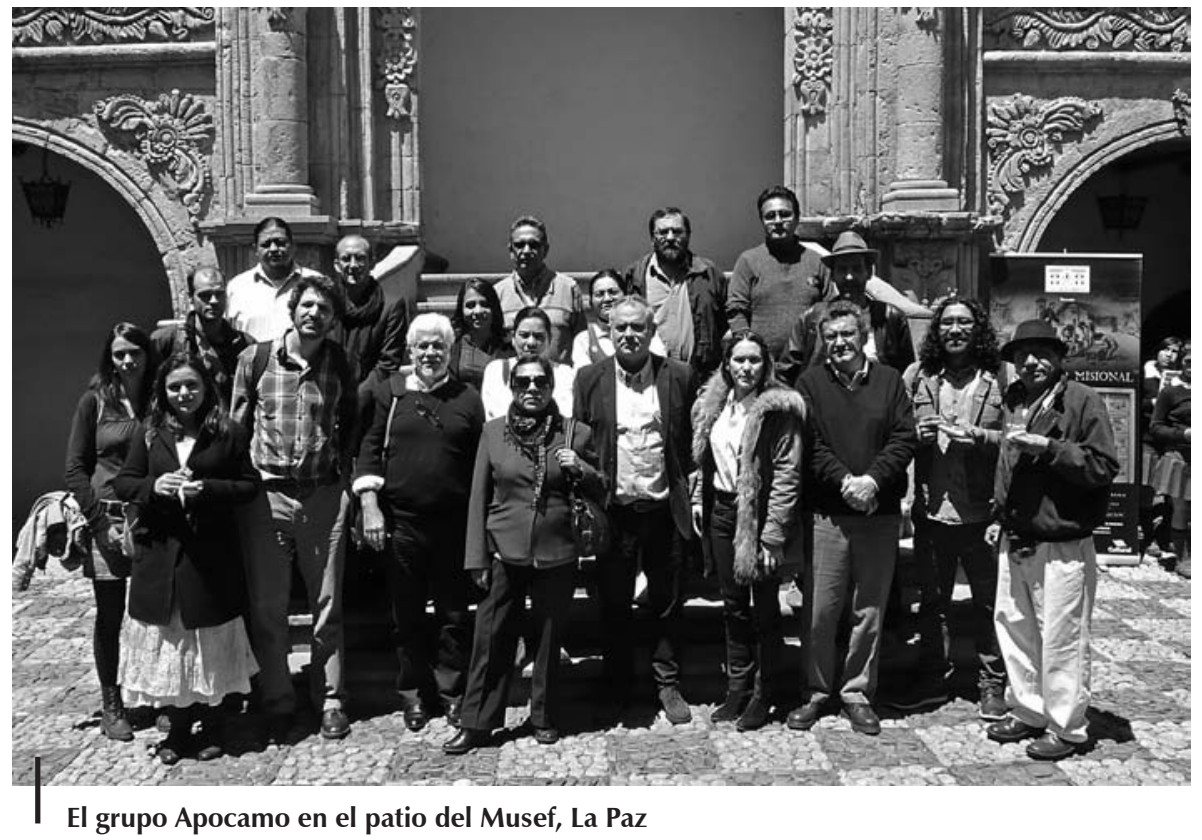

reflexión sobre la aplicación del llamado derecho consuetudinario reconocido por el sistema jurídico nacional boliviano. Describió, en particular, el caso de una acusación de brujería que se saldó con el entierro vivo del acusado, un refugiado de origen brasileño. Philippe Erikson constata que esta ejecución no tenía ningún fundamento antropológico. Añade que es difícil pensar en la existencia de un modelo de legalidad «chacobo» equivalente a la legalidad boliviana, entre otras cosas por la importancia del registro escrito jurisprudencial inherente a esta última. El ponente agregó que buscar un modelo legal «chacobo» e intentar describirlo es posiblemente una deriva etnocéntrica. La presentación concluyó señalando que, después de todo, el acusado murió no tanto por una supuesta ley chacobo, sino por la ley trágica de un destino ineludible. Oscar Espinosa y Ana María Lema propusieron algunos alcances antes de dejar paso a las preguntas del público.

Después y con el título «iPlumas arriba! Reformas políticas estatales y nueva figura del poder en el pueblo yuqui», David Jabin examinó también un caso situado en Bolivia, en el año 2010, cuando los pueblos indígenas de las tierras bajas procedieron a elegir los representantes indígenas que integrarían las asambleas legislativas de siete departamentos, en el marco de la nueva Constitución Política de 2009. Unos años después de la puesta en marcha de esta reforma dos preguntas surgen según David Jabin. En primer lugar ¿̇cuáles son los efectos locales de estas políticas estatales?; y por otro lado ¿cuáles los cambios en la formas de acceso y ejercicio del poder tradicional provocados al interior de tales sociedades indígenas? A través de una reflexión antropológica sobre el pueblo Yuqui, David Jabin propuso algunos elementos de respuesta a tales interrogantes. Jean-Pierre Chaumeil y Jorge Komadina ofrecieron a continuación algunos comentarios. 
Después de las intervenciones del público, Elías Caurey cerró la tarde de ponencias sobre Bolivia con una presentación titulada Autonomía indígena y educación: los currículos regionalizados en Bolivia. Esta ponencia describió los alcances que tiene la Autonomía Indígena Originaria Campesina (AIOC) de acuerdo a la legislación boliviana en cuanto al tema educativo. A través del currículo regionalizado, considerando su carácter intracultural, se describió el rol de los Consejos Educativos de Pueblos Originarios de Bolivia en la construcción de las propuestas educativas desde la visión de las Naciones y Pueblos Indígena Originarios (NPIOS). Se examinó también las bondades que brinda la AIOC y el currículo regionalizado para buscar la autodeterminación de las Niops y los peligros que implicaría si no se lo encara como debe de ser el proceso. Gabriela Canedo y François Correa estuvieron a cargo de los comentarios antes de dejar paso al debate plenario. Unas palabras finales de los organizadores dieron paso al Brindis de clausura.

Por la mañana del día 18 de octubre, y a puerta cerrada, tuvo lugar la reunión interna de los miembros del GRDI «Apocamo» en cuyo orden del día figuraba la preparación del próximo seminario-taller así como el examen de solicitud de nuevos miembros. Una vez terminada la reunión, los miembros de Apocamo presentes agradecieron a la Sra. Elvira Espejo Ayca, directora del Musef, la acogida y hospitalidad brindada por su institución, para dirigirse luego a una visita guiada de las muestras temporales que el Musef presenta en estos días. 